\title{
New Generation Pharmacogenomic Tools: A SNP Linkage Disequilibrium Map, Validated SNP Assay Resource, and High-Throughput Instrumentation System for Large-Scale Genetic Studies
}

Francisco M. De La Vega, David Dailey, Janet Ziegle, Julie Williams, Dawn Madden, and Dennis A. Gilbert Applied Biosystems, Foster City, CA, USA

\begin{abstract}
Since public and private efforts announced the first draft of the human genome last year, researchers have reported great numbers of single nucleotide polymorphisms ( $S N P$ S). We believe that the availability of wellmapped, quality SNP markers constitutes the gateway to a revolution in genetics and personalized medicine that will lead to better diagnosis and treatment of common complex disorders. A new generation of tools and public SNP resources for pharmacogenomic and genetic studies-specifically for candidate-gene, candidate-region, and whole-genome association studies-will form part of the new scientific landscape. This will only be possible through the greater accessibility of SNP resources and superior highthroughput instrumentation-assay systems that enable affordable, highly productive large-scale genetic studies. We are contributing to this effort by developing a high-quality linkage disequilibrium SNP marker map and an accompanying set of ready-to-use, validated SNP assays across every gene in the human genome. This effort incorporates both the public sequence and SNP data sources, and Celera Genomics' human genome assembly and enormous resource of physically mapped SNPs (approximately 4000000 unique records). This article discusses our approach and methodology for designing the map, choosing quality SNPs, designing and validating these assays, and obtaining population frequency of the polymorphisms. We also discuss an advanced, high-performance SNP assay chemistry-a new generation of the TaqMan ${ }^{\circledR}$ probe-based, 5' nuclease assay-and highthroughput instrumentation-software system for large-scale genotyping. We provide the new SNP map and validation information, validated SNP assays and reagents, and instrumentation systems as a novel resource for genetic discoveries.
\end{abstract}

\section{INTRODUCTION}

The promise of personalized medicine is that genetic differences between individuals will predict disease risk and response to therapies and improve medical treatment. The availability of human genome data $(11,21)$, and the accompanying deluge of putative single nucleotide polymorphisms (SNPs) (3), is enabling the large-scale studies necessary to achieve these goals.
However, having SNP databases is only the first requirement for conducting genetic association studies. Putative polymorphisms must be validated (15) and assembled into a standardized SNP marker map (19). In addition, SNP information must be easily accessible, and standardized assay reagents must be developed, validated, and made available to enable the high throughput and automation necessary to screen many SNPs on many individuals (19). The challenge is to accomplish all this while reducing the cost per genotype (i.e., study cost), and the required completion time for the entire study, enough to make large-scale studies feasible.

To address these needs, Applied Biosystems is creating a reference SNP set based on SNP and genomic information from both proprietary and public databases. Researchers can use this set and its accompanying validated, ready-to-use assays and reagents for linkage disequilibrium mapping. The set provides high-density coverage of all known gene regions to enable easier and more affordable candidate gene association studies and candidate region association studies. With access to this resource, researchers can select SNPs across candidate genes or chromosomal regions that are most suitable for a given study, and quickly translate that information into practice by directly obtaining assay protocols and reagents for those SNPs. This resource has the potential to become a "core" set of SNPs and the TaqMan ${ }^{\circledR}$ probe-based assay reagents can be expanded or refined as additional information, such as haplotype definition for some or all of the genome, becomes available. This SNP set is also being used to develop panels of chromosome-based assays for cost-efficient whole genome association (WGA) studies using the oligonucleotide ligation assay (OLA) (6) with highly parallel array readout technology.

\section{BUILDING A LINKAGE DISEQUILIBRIUM SNP MARKER SET}

Linkage disequilibrium (LD) is the nonrandom association of alleles in a chromosomal segment and constitutes the basis of 
all genetic mapping. Selecting SNPs as genetic markers for LD studies involves considering all genetic and assay-specific technical factors that affect the ability to find association between a marker and the susceptibility mutations being mapped.

The extent of LD across a genomic region dictates the SNP density necessary to ensure association between a marker and the causative allele sought. Early attempts to model the extent of LD predicted very short LD of only a few kilobases (9). However, recent empirical surveys report average LD levels between 5 and $60 \mathrm{~kb}$, and extending up to hundreds of kilobases $(1,5,17)$, which implies that the number of SNPs required for WGA studies could range from 50000 to 250000 , and that markers spaced by tens of kilobases will suffice for candidate gene studies. Common SNPs are the most likely to be useful for LD studies across more than one population since they represent ancient mutations that arose before ethnic group segregation (18), and simulation studies (16) suggest that they are more likely than cSNPs to be in LD with a given causative allele regardless of whether the allele is present at low or high frequency.

In assembling our SNP set, we are focused on common SNPs in a hybrid gene-based approach. SNPs are considered "common" when the minor allele frequency is $>15 \%$ in at least one of the populations used for validation. Currently, the gene list we use includes 25083 gene regions derived by Celera Genomics. We define a "gene region" as bounded by the first and last transcribed base, including untranslated regions, plus $10 \mathrm{~kb}$ upstream and downstream to account for uncharacterized exons and regulatory regions. Selecting SNPs within gene regions, at an average density of one per $10 \mathrm{~kb}$, makes the map resemble a gene-focused picket fence. Density for specific regions is adjusted as data on recombination (4) and LD extent (5) emerges. At the close of the project we may elect to complement this set with additional SNPs in intergenic regions, in particular noncoding regions of homology between mouse and human (12).

\section{SNP ASSAY DEVELOPMENT AND VALIDATION}

More than 2000000 SNPs reside in public databases (20). Celera Genomics discovered more than 2000000 additional high-quality putative SNPs by sequencing fragments from five donors of different ethnic backgrounds during the assembly of the human genome (21). Additionally, Celera has mapped most public SNPs onto the Celera assembly (8). Together these represent a resource of more than 4000000 unique SNPs that we leveraged to construct the LD SNP set (Celera RefSNP database version 3.4.1).

Literature reports that as few as 50\% of SNPs randomly selected from the public databases are polymorphic and yield working assays $(3,15)$. Thus, it is important to enhance the likelihood of obtaining a validated SNP assay at the end of the process by defining prioritization criteria. A key criterion for candidate SNPs to advance in our process is evidence of independent discovery of the minor allele. Cross-references between Celera SNPs and those available in public sources are used to satisfy this requirement. Additionally, for Celera SNPs with no equivalent in the public, we require observation of the minor allele in reads from two independent donors. Finally, for Celera SNPs where the minor allele was found in only one donor, often we can find an independent instance of the minor allele by searching the consensus assembly of the public Human Genome Project (11). Currently this selected SNP pool includes about 1000000 SNPs. This "triage" procedure has led to extremely high validation rates and highly heterozygous SNPs (see example below for chromosome 22).

We have developed a multi-step, high-throughput assay design pipeline to ensure optimum performance of the resulting assays. Our goal is to enable automation, minimize assay failure, and ensure compatibility of the SNP sequence with our current TaqMan probe-based $5^{\prime}$ nuclease chemistry and future assay formats. A stringent scoring system is used to select only those SNP context sequences with the highest probability of success. Details of the bioinformatics pipeline will be reported elsewhere, but a brief account of the steps follows (Figure 1).

The first step of the pipeline involves masking adjacent SNPs to the target polymorphism and any sequence discrepancy between the Celera and the HGP human genome assembly, in the 600 bases of context sequence. This prevents primers and probes from being placed on top of other SNPs and maximizes the chance that they will hybridize to the correct genomic sequence.

TaqMan 5' nuclease primers and probes are designed using a proprietary algorithm developed for our Assays-by-Design ${ }^{\mathrm{SM}}$ custom oligonucleotide reagent service. Oligonucleotides are designed in batch mode without manual intervention, and a scoring scheme selects the best sequences for a given SNP. The design algorithm implements the thermodynamic and heuristic

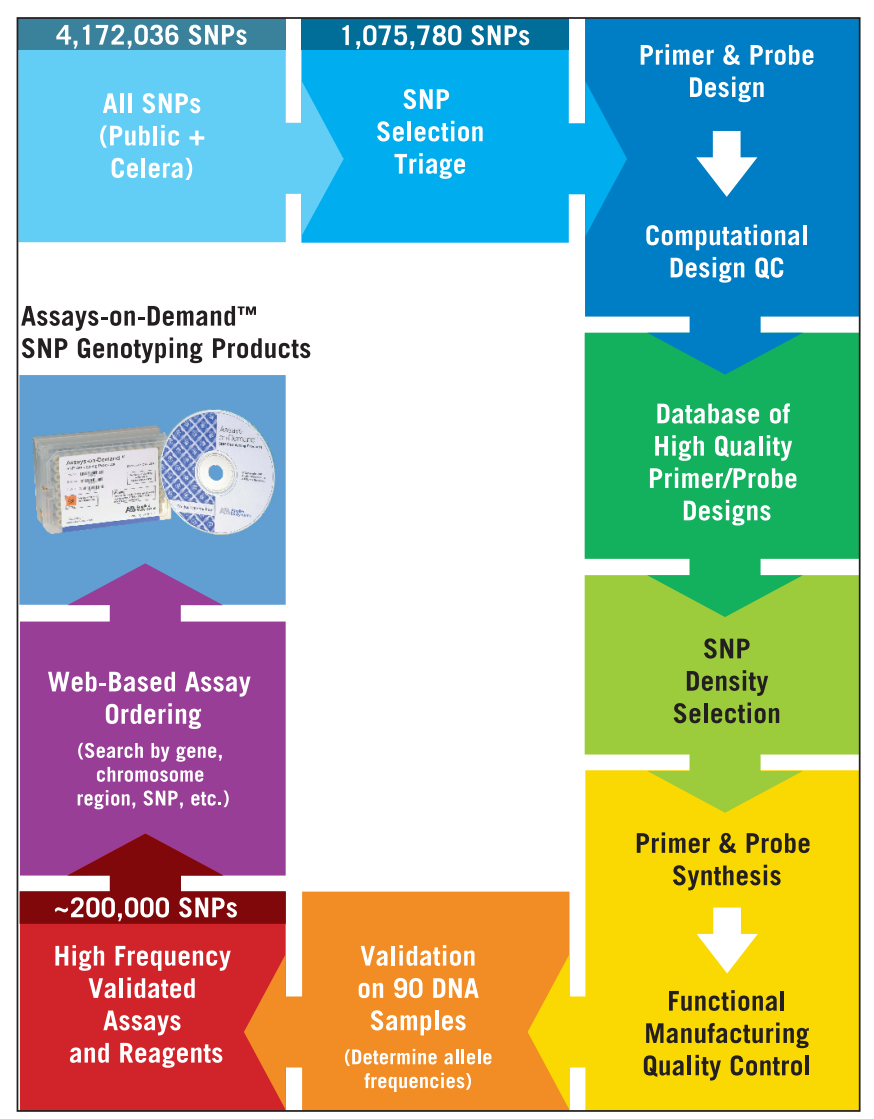

Figure 1. Development pipeline for $200 \mathrm{~K}$ SNP map and validated assays. 
rules described in our $5^{\prime}$ nuclease assay design guidelines (13), plus additional empirically derived factors that increase manufacturability and assay performance. At this point, we can design probes successfully for $97 \%$ of SNPs. After this step, a further computational quality-control step is also performed in the context of the genome, which allows us to eliminate potentially problematic SNP targets that may arise from repeated genomic regions, pseudo-SNPs, and other possible assembly artifacts (7).

Finally, the primers and probes are synthesized, and two additional quality-control steps occur. The first tests oligonucleotide integrity, and the second tests assay performance against a panel of 10 DNA samples. Only assays that pass this manufacturing $\mathrm{QC}$ are moved on for validation in the population panels.

Assay validation in population samples ensures that the locus is polymorphic and that the allele frequency will be adequate for association studies in a variety of populations. We obtained 90 samples from the Coriell Human Variation Collection (7). By obtaining individual genotypes from a panel of 45 African Americans, a panel of 45 Caucasians, and a chimp DNA sample (to provide insight into ancestral alleles), we will obtain sufficient information to estimate linkage disequilibrium between SNPs in the map and to computationally infer common haplotypes. This will provide additional information on the usefulness of the markers and the coverage provided for a given study, and provide an independent assessment of assay performance.

The performance of each assay is benchmarked against stringent criteria for background signal (e.g., low signal in the control experiments run without template), adequate signal generation (e.g., good separation between control experiments run without template and allele clusters), and specificity. We require a maximum of three clusters, and at least two clusters must be observed to pass assay. Lastly, we specify that at least $90 \%$ of samples yield callable genotypes.

Using chromosome 22 as a pilot project, we validated 2260 SNP assays, confirming that $94 \%$ of the SNPs tested with the population panels were polymorphic and $90 \%$ of the assays passed our stringent performance criteria. When using a minor allele frequency cut-off of $15 \%$ or greater, $83 \%$ of the samples from the African-American panel and $88 \%$ from the Caucasian panel meet the criteria. Ninety-seven percent $(97 \%)$ of SNPs have a minor allele frequency of $15 \%$ or greater in at least one of the population panels. Ninety-four percent $(94 \%)$ of samples achieved a minor allele frequency of $20 \%$ or greater in at least one population tested, and $60 \%$ of samples showed a minor allele frequency of $20 \%$ or greater in both populations tested. These figures represent an extremely high SNP validation rate and an unprecedented yield of common SNPs useful in LD mapping $(15,19)$. We are currently analyzing the extent of LD among these markers, as well as surveying the common haplotype blocks across the gene regions, to better document the utility of the SNPs selected for the set. The results of these analyses for a set of chromosomes that we have prioritized in our pipeline will be published elsewhere.

To address our automation goal, the validation lab has implemented auto allele calling software, with the goal to automatically analyze at least $90 \%$ of validated assay data without user intervention. Thus, the validation process provides a testing ground for the software, and accelerates development of a commercially available version.

\section{IMPROVEMENTS IN THE ASSAY CHEMISTRY}

To meet high-throughput demands, the SNP assays and reagents must rely on simple, easily automated reaction chemistry and be compatible with readily available high-throughput instrumentation and software systems. For example, they must have few enzymatic steps, no post-reaction transfer of liquids, and universal reaction conditions to make robotic liquid handling automation easy. In addition, the assays, reagents, and high-throughput workflow must be easy for researchers to implement and automate, relying on components that come ready to use and require no optimization.

The TaqMan probe-based $5^{\prime}$ nuclease assay chemistry meets these requirements and unites PCR amplification and signal generation into a single step, simplifying automation of both reaction set-up and data collection. In this system a hybridization probe with fluorogenic and quencher tags is cleaved by the $5^{\prime}$ nuclease activity of Taq DNA polymerase during PCR amplification. Cleavage produces fluorescence by freeing the fluorogenic molecule from the quencher. By using two probes, one specific to each allele of the SNP and labeled with distinct fluorogenic tags, both alleles are specifically detected in a single tube (13). In addition, the fluorescence $5^{\prime}$ nuclease assays are part of an easy-to-use, automated system for SNP genotyping. Figure 2 provides an overview of the TaqMan probe-based $5^{\prime}$ nuclease assay chemistry for SNP genotyping.

This system was first adapted to allelic discrimination in 1998 (13) and has since been modified for high-throughput SNP genotyping purposes. Chemistry improvements have increased assay design flexibility, enabled easy protocol standardization, enabled the use of universal reagents, and reduced background fluorescence-all requirements for high throughput SNP processing and allelic discrimination.

Previously, for example, probes of up to 30 bases were required to achieve the specificity required for scoring SNPs. The conjugation of a DNA minor groove binder (MGB) to the probe significantly stabilizes probe-template complexes (10), enabling the use of probes in the 13- to 20-mer size range. These probes display better mismatch discrimination, are easier to design for challenging genetic regions such as those high in GC content or those in variable context sequences, and increase the signal-to-noise ratio by bringing the quencher closer to the fluorescent tag. Most significantly for high-throughput SNP scoring, they increase the melting temperature window that can be used in reaction protocols, which allows all the SNP assays to run under identical conditions.

Early $5^{\prime}$ nuclease designs used the quencher TAMRA ${ }^{\mathrm{TM}}$ (Applied Biosystems). However, because TAMRA emits its own fluorescence, it complicated signal detection. A new non-fluorescent quencher, because it does not fluoresce upon absorption of the fluorescence resonance energy from the fluorescent tag, provides improved signal detection $(2,14)$ and facilitates automated allele calling - another requirement for high-throughput SNP scoring.

Often in a pharmacogenomic study, the most precious reagent is the DNA sample itself. High-quality DNA quantification using real-time analysis on the ABI PRISM ${ }^{\circledR} 7900 \mathrm{HT}$ Sequence Detection System with the TaqMan RNase P Control Reagents Kit permits optimal amounts of DNA per reaction to 
maximize study efficiency. The $7900 \mathrm{HT}$ system uses a $5-\mu \mathrm{L}$ reaction volume and typically consumes 1 ng of DNA per genotyping reaction, which minimizes reagent costs and conserves DNA template samples.

The $5^{\prime}$ nuclease assay is ideally suited for automation by virtue of its easy, three-step workflow. A universal master mix, including probes and primers, can be added directly to plates of dry or fresh DNA using standard robotics. Plates are sealed and cycled using standard thermal cyclers, such as the Applied Biosystems Dual 394-Well GeneAmp ${ }^{\circledR}$ PCR System 9700 thermal cycler. Following cycling, plates are automatically read on the $7900 \mathrm{HT}$, which supports the collection of more than 250000 genotypes per day. In addition, the availability of thermal cyclers with automated lid handling can increase throughput by enabling robotics integration for 24-hour unattended operation. Automation software is also available to increase both quality and throughput. For example, automation of allele calling can remove inter-technician variability, increasing confidence in data quality, and reduce the time spent on data analysis by 8.5 person-hours per day.

\section{MAKING THE RESOURCE AVAILABLE TO RESEARCHERS}

Access to SNP information from both the Celera RefSNP database and public SNP databases was a significant factor in the success of LD SNP map development, and in efficiently conducting validation studies. Although researchers have made significant progress in developing, curating, and annotating in- dividual databases that leverage public and private genomic information, ours is the first resource that allows them to leverage all 4000000 currently known SNPs and maintain direct access to assays and reagents that enable association studies.

To facilitate use of the resource and optimize access to SNP assays, we are developing a Web interface that will let users research and select assays and reagents based on a wide variety of criteria. For example, researchers who know the exact SNPs they want can access them using identifiers (e.g., Celera variation ID, $\mathrm{dbSNP}$ rs or ss ID). Investigators can also research SNPs by gene name (e.g., HUGO gene symbol, RefSeq ID, Celera transcript ID), or by location within a particular genome location or reference marker range (e.g., microsatellite, sequence-tagged site) they are interested in. Within these regions, the user can specify filtering criteria based on SNP distribution density, SNP type (e.g., intronic, coding), a user-specified flanking region, a specific allele frequency, or gene overlap.

After ordering through the Web-based Applied Biosystems interface, researchers will receive additional information that will let them perform the assay (e.g., detection instrumentation parameters), and fully integrate the SNP into their studies (e.g., context sequence, allele-dye key, SNP type, allele frequency).

\section{CONCLUSIONS AND FUTURE DIRECTIONS}

Integrating information from both public and private human genome efforts, we have created a high-quality LD map of validated SNPs. Expertise in assay design and bioinformatics has allowed us to turn this information resource into a set of

\section{Part A}

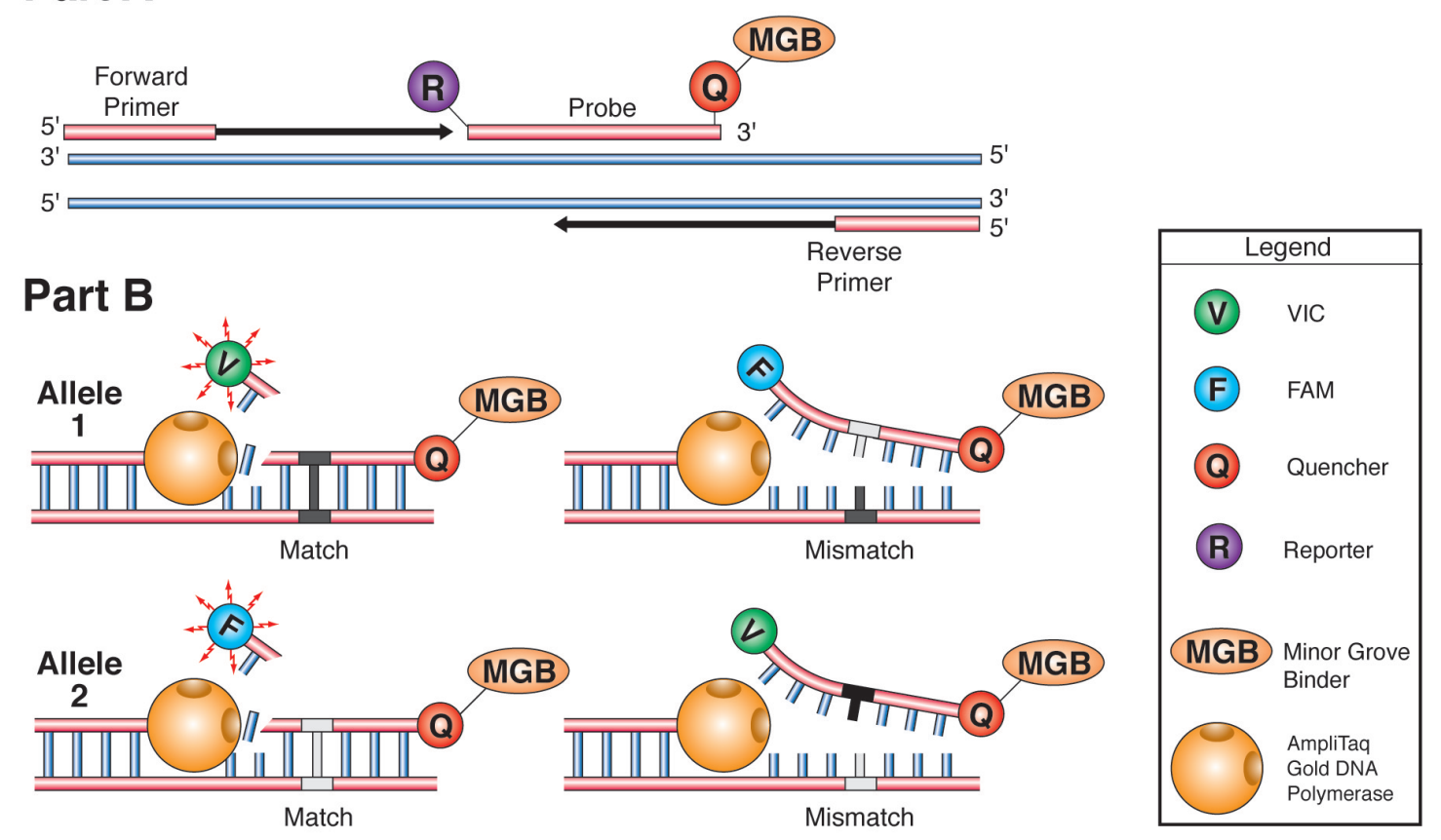

Figure 2. TaqMan probe-based 5' nuclease assay chemistry for SNP genotyping. 
over 200000 validated, easy-to-use, individual SNP assays and TaqMan ready-to-use assay reagents. Recent enhancements of the $5^{\prime}$ nuclease chemistry, such as the addition of a minor groove binder and a nonfluorescent quencher, and its integration with an automated detection system, the $7900 \mathrm{HT}$, have ensured the success of our efforts. To provide access to these tools to the research community, we are developing a Webbased bioinformatics and ordering system where they can easily search for SNPs and order assay reagents, thus reducing the time and costs associated with candidate-gene and candidate-region association studies. Improvements in the automation workflow and cost efficiency of the $5^{\prime}$ nuclease assay and instrumentation are continuing. For example, new formats in development that will enable the use of even smaller volumes of samples and reagents will make pharmacogenomic studies even easier to implement and afford.

Besides enabling candidate-gene and candidate-region association studies on the $5^{\prime}$ nuclease system, the LD map is also being implemented on an ultrahigh-throughput SNP genotyping platform currently under development for researchers who want to undertake WGA studies. This system will leverage the specificity of the OLA-PCR assay chemistry and the highly parallel detection of Illumina's BeadArray ${ }^{\mathrm{TM}}$ technology (San Diego, CA, USA). The system will enable the generation of approximately 2100000 genotypes per 24 hours, and all components of the assay are universal except for the SNP-specific OLA probes.

Moving forward, we are rapidly designing assays for over 4000000 SNPs from the Celera database that will cover every gene in the human genome. As these SNPs progress through the assay development pipeline, we expect to identify many with the necessary variability for genetic association studies. These SNPs will be turned into additional assays for the $5^{\prime}$ nuclease system and made available for ordering. Furthermore, we imagine that as SNPs in the collection become increasingly characterized, they will be grouped together into convenient SNP sets optimized for specific assays such as p450 genotyping and diseasespecific gene studies.

\section{ACKNOWLEDGMENTS}

We are indebted to Hadar Avi-Itzhak, Ryan Koehler, Charles Scafe, Eugene Spier, Xiaoping Su, Yu Wang, and Marion Webster for their contributions to the development of the bioinformatics pipeline. The authors thank Jinghui Zhang and Frank Liu for performing custom queries and processing the JSNP database through their data integration pipeline, and Emily Winn-Deen for genotyping some samples in her lab, all from Celera Genomics. Thanks are also due to Andrew Clark (U. Penn.), Kenneth Kidd (Yale Medical School), and Kit Lau and John Sninsky (Celera Diagnostics) for many helpful discussions, and Mignon Fogarty for assistance with the manuscript.

\section{REFERENCES}

1.Abecasis, G.R., E. Noguchi, A. Heinzmann, J.A. Traherne, S. Bhattacharyya, N.I. Leaves, G.G. Anderson, Y. Zhang, et al. 2001. Extent and distribution of linkage disequilibrium in three genomic regions. Am. J. Hum. Genet. 68:191-197.
2.Afonina, I.A., M.W. Reed, E. Lusby, I.G. Shishkina, and Y.S. Belousov. 2002. Minor groove binder-conjugated DNA probes for quantitative DNA detection by hybridization-triggered fluorescence. BioTechniques 32:940949 .

3.Altshuler, D., V.J. Pollara, C.R. Cowles, W.J. Van Etten, J. Baldwin, L. Linton, and E.S. Lander. 2000. An SNP map of the human genome generated by reduced representation shotgun sequencing. Nature 407:513-516.

4.Collins, A., S. Ennis, P. Taillon-Miller, P.Y. Kwok, and N.E. Morton. 2001 Allelic association with SNPs: metrics, populations, and the linkage disequilibrium map. Hum. Mutat. 17:255-262.

5.Daly, M.J., J.D. Rioux, S.F. Schaffner, T.J. Hudson, and E.S. Lander. 2001. High-resolution haplotype structure in the human genome. Nat. Genet. 29:229-232.

6.Favis, R., J.P. Day, N.P. Gerry, C. Phelan, S. Narod, and F. Barany. 2000. Universal DNA array detection of small insertions and deletions in BRCA1 and BRCA2. Nat. Biotechnol. 18:561-564.

7.Heil, J., S. Glanowski, J. Scott, E. Winn-Deen, I. McMullen, L. Wu, C. Gire, and A. Sprague. An automated computer system to support ultra high throughput SNP genotyping, p. 30-40. In Pacific Symposium on BioComputing, Lihue, Hawaii, January 3-7, 2002.

8.Kerlavage, A., V. Bonazzi, M. di Tommaso, C. Lawrence, P. Li, F. Mayberry, R. Mural, M. Nodell, et al. 2002. The Celera Discovery System. Nucleic Acids Res. 30:129-136.

9.Kruglyak, L. 1999. Prospects for whole-genome linkage disequilibrium mapping of common disease genes. Nat. Genet. 22:139-144.

10.Kutyavin, I.V., I.A. Afonina, A. Mills, V.V. Gorn, E.A. Lukhtanov, E.S. Belousov, M.J. Singer, D.K. Walburger, et al. 2000. 3'-minor groove binderDNA probes increase sequence specificity at PCR extension temperatures. Nucleic Acids Res. 28:655-661.

11.Lander, E.S., L.M. Linton, B. Birren, C. Nusbaum, M.C. Zody, J. Baldwin, K. Devon, K. Dewar, et al. 2001. Initial sequencing and analysis of the human genome. Nature 409:860-921.

12.Levy, S., S. Hannenhalli, and C. Workman. 2001. Enrichment of regulatory signals in conserved non-coding genomic sequence. Bioinformatics 17:871877.

13.Livak, K.J. 1999. Allelic discrimination using fluorogenic probes and the 5' nuclease assay. Genet. Anal. 14:143-149.

14.Lukhtanov, E.L., M. Metcalf, and M.W. Reed. 2001. Fluorogenic DNA Probes for multicolor hybridization assays. Am. Biotechnol. Lab. 19:68-69.

15.Marth, G., R. Yeh, M. Minton, R. Donaldson, Q. Li, S. Duan, R. Davenport, R.D. Miller, and P.Y. Kwok. 2001. Single-nucleotide polymorphisms in the public domain: how useful are they? Nat. Genet. 27:371-372.

16.Nordborg, M. and S. Tavare. 2002. Linkage disequilibrium: what history has to tell us. Trends Genet. 18:83-90.

17.Reich, D.E., M. Cargill, S. Bolk, J. Ireland, P.C. Sabeti, D.J. Richter, T. Lavery, R. Kouyoumjian, et al. 2001. Linkage disequilibrium in the human genome. Nature 411:199-204.

18.Reich, D.E. and E.S. Lander. 2001. On the allelic spectrum of human disease. Trends Genet. 17:502-510.

19.Remm, M. and A. Metspalu. 2002. High-density genotyping and linkage disequilibrium in the human genome using chromosome 22 as a model. Curr. Opin. Chem. Biol. 6:24-30.

20.Sachidanandam, R., D. Weissman, S.C. Schmidt, J.M. Kakol, L.D. Stein, G. Marth, S. Sherry, J.C. Mullikin, et al. 2001. A map of human genome sequence variation containing 1.42 million single nucleotide polymorphisms. Nature 409:928-933.

21.Venter, J.C., M.D. Adams, E.W. Myers, P.W. Li, R.J. Mural, G.G. Sutton, H.O. Smith, M. Yandell, et al. 2001. The sequence of the human genome. Science 291:1304-1351.

\section{Address correspondence to:}

Dr. Francisco De La Vega

Applied Biosystems

850 Lincoln Centre Drive

Foster City, CA 94404, USA

e-mail:delavefm@appliedbiosystems.com 